\title{
Compact Wakeup Scheduling in Wireless Sensor Networks
}

\author{
Junchao Ma and Wei Lou \\ Department of Computing, \\ The Hong Kong Polytechnic University, Kowloon, Hong Kong \\ $\{$ csjma, csweilou\}@comp.polyu.edu.hk
}

\begin{abstract}
Wakeup scheduling has been widely used in wireless sensor networks (WSNs), for it can reduce the energy wastage caused by the idle listening state. In a traditional wakeup scheduling, sensor nodes start up numerous times in a period, thus consuming extra energy due to state transitions (e.g. from the sleep state to the active state). In this paper, we address a novel interference-free wakeup scheduling problem called compact wakeup scheduling, in which a node needs to wake up only once to communicate bidirectionally with all its neighbors. However, not all communication graphs have valid compact wakeup schedulings, and it is NP-complete to decide whether a valid compact wakeup scheduling exists for an arbitrary graph. To tackle this problem, we define the compact wakeup deficiency of a graph to extend the scheduling to general graphs. In particular, tree and grid topologies, which are commonly used in WSNs, have valid compact wakeup schedulings. We propose polynomial-time algorithms using the optimum number of time slots in a period for trees and grid graphs. Simulations further validate our theoretical results.
\end{abstract}

Keywords: energy efficiency, compact wakeup scheduling, wireless sensor networks.

\section{INTRODUCTION}

Wireless sensor networks (WSNs) consist of hundreds to thousands of tiny, inexpensive and battery-powered wireless sensing devices which organize themselves into multi-hop radio networks. As the batteries of most sensor nodes are non-rechargeable, one key challenging issue is to schedule the activities of nodes to minimize the energy consumption. The major source of energy wastage [1], [2] in WSNs is the idle listening state in the radio modules, which in fact 
consumes almost as much energy as receiving. Therefore, nodes are generally scheduled to sleep when the radio is not in use [3], and wake up when necessary. By using wakeup scheduling, nodes could operate in a low duty cycle mode, and periodically start up to check the channel for activity.

The previous studies [4], [5] in the wakeup scheduling did not, however, consider all possible energy consumption, especially the energy consumed in the state transitions, for example, from the sleep state to the listening state or transmitting state. After such a scheduling, a node may start up numerous times in a period to communicate with its neighbors. Note that the typical startup time is on the order of milliseconds, while the transmission time may be less than the startup time if the packets are small [6]. Take Tmote Sky [7] as an example, the time and energy consumption to activate a node is about $1.4 \mathrm{~ms}$ and $17 \mu \mathrm{J}$, respectively; whereas the time and energy consumption to transmit 1 byte is about $0.032 \mathrm{~ms}$ and $1.7 \mu \mathrm{J}$, respectively. If a sensor node starts up too frequently, it not only needs extra time, but also consumes extra energy for state transitions. Moreover, it reduces the battery capacity due to the current surges in the state transitions.

Fig. 1 shows the battery voltage of Tmote Sky sensors with different startup frequencies but with the same duty cycle (50\%): One starts up every $20 \mathrm{~ms}$, stays in the receive state for $10 \mathrm{~ms}$ and turns to the sleep state for the rest period; the other one starts up every $100 \mathrm{~ms}$, stays in the receive state for $50 \mathrm{~ms}$ and turns to the sleep state for the rest period. We can see that about $8 \%$ battery voltage can be saved by reducing the startup frequency from five times to once in every $100 \mathrm{~ms}$. Therefore, the state transitions should be considered in the wakeup scheduling design.

In [8], energy efficient centralized and distributed algorithms are proposed to reduce the frequency of state transitions of each node to twice in a data gathering tree: once for receiving data from its children, and once for sending data to its parent. If the network topology is a directed acyclic graph (DAG) where each node $v_{i}$ has $k_{i}$ parents, the scheduling in [8] would require $v_{i}$ to wake up $k_{i}+1$ times as the parent nodes are not scheduled together. Moreover, the two-way (or bidirectional) communication is not taken into consideration. An interesting problem is to design an efficient scheduling where a node could wake up only once and finish all communication tasks with its neighbors consecutively and bidirectionally.

In this paper, we propose compact wakeup scheduling, a novel time division multiple access (TDMA) approach to the wakeup scheduling problem, to minimize the frequency of state 


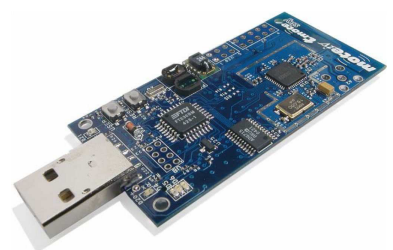

(a) Tmote Sky

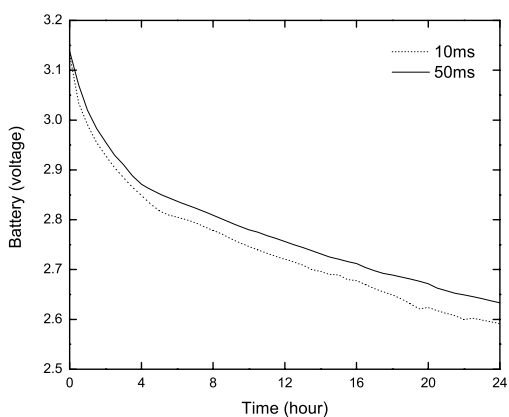

(b) Battery

Fig. 1: (a) Tmote Sky sensor. (b) The battery voltage of Tmotes with different startup frequency. AA Carbon-Zinc batteries are used in the experiment. 10ms and $50 \mathrm{~ms}$ are active time in each period.

transitions. Compact wakeup scheduling assigns consecutive time slots to all the links incident to a node $v_{i}$ so that $v_{i}$ can start up only once to communicate bidirectionally with all its neighbors in one scheduling period $T$.

Apart from reducing the transient time and energy cost in the state transitions, compact wakeup scheduling also has other benefits. The network delay, which is a major concern in time-critical monitoring systems like [9], can be reduced. For instance, a sensor may need to wait until all its neighbors wake up so that it can collect the real time data from these neighbors to make the local computation on these data. Note that, compact wakeup scheduling can not only reduce the state transitions of transceivers, but also reduce the state transitions of other components in the nodes, such as external memory and sensing devices.

The main contributions of this paper are summarized as follows. (1) We formulate the compact wakeup scheduling problem in WSNs to minimize the frequency of state transitions, and prove it to be NP-complete. (2) We present polynomial-time algorithms using the optimum number of time slots in a period for trees and grid graphs. In grid graphs, we point out all the possible coloring patterns and give the lower bound as well as the upper bound of the compact wakeup scheduling. (3) We define the compact wakeup deficiency of a graph in the case of graphs without valid compact wakeup schedulings. (4) We develop simulations to show the efficiency of compact wakeup scheduling. 
The rest of this paper is organized as follows. Section II reviews the related work. Section III describes the system model and formulates the compact wakeup scheduling problem. Section IV presents polynomial-time algorithms for trees and grid graphs. Section V defines the compact wakeup deficiency for graphs without valid compact wakeup schedulings. Section VI shows the performance evaluation. Section VII concludes the paper and provides directions for future research.

\section{RELATED WORK}

Wakeup scheduling has attracted a lot of interest in WSNs in virtue of its energy efficiency. S-MAC [1] is a contention-based MAC scheme. In S-MAC, nodes periodically sleep and wake up, and each active period is of a fixed size with a variable sleep time. T-MAC [2] improves S-MAC by adopting a dynamic duty cycle, i.e., transmitting all messages in bursts and ending the listening period when nothing is heard within a limited time. DW-MAC [3] allows nodes to wake up on demand during the sleep period and ensures that data transmissions do not collide at their intended receivers. TreeMAC [10] is a localized TDMA MAC protocol, which is designed to achieve high throughput and low congestion with low overhead.

Link scheduling is time slot assignments to communication links in TDMA MAC protocols. Ramanathan and Lloyd [11] consider both the tree networks and arbitrary networks, and the performance of the proposed algorithms is bounded by the thickness of a network. In [12], Gandham et al. propose a link scheduling algorithm involving two phases. In the first phase, a valid edge coloring is obtained in a distributed fashion. In the second phase, each color is mapped to a unique time slot, and the hidden terminal problem as well as the exposed terminal problem is avoided by assigning each edge a direction of transmission. The overall scheduling requires at most $2(\Delta+1)$ time slots when the topologies are acyclic, where $\Delta$ is the maximum degree of a graph. In [5], Wang et al. propose a degree-based heuristic algorithm with performance guarantee to obtain a good interference-free link scheduling to maximize the throughput of the network. In the algorithm, the sensors are scheduled individually in a predefined order without consecutive assignment of time slots, and each node is assigned the best possible time slot to transmit or receive without causing interferences to the already-scheduled sensors. In [13], Wu et al. propose efficient centralized and distributed scheduling algorithms that reduce the energy cost of state transitions, and also propose an efficient method to construct an energy-efficient 
data gathering tree. In [8], Ma et al. address the contiguous link scheduling problem by applying the interval vertex coloring in a merged conflict graph, and assigning consecutive time slots to the links incident to one node to achieve better energy efficiency.

\section{PROBLEM Formulation}

In this section, we first present the system model, then formulate the compact wakeup scheduling problem.

\section{A. System Model}

We assume that a WSN has $n$ static sensor nodes equipped with single omni-directional antennas, and all the nodes have the same communication range. The network is represented as a communication graph $G=(V, E)$, where $V=\left\{v_{1}, v_{2}, \cdots, v_{n}\right\}$ denotes the set of nodes, and $E=\left\{e_{1}, e_{2}, \cdots, e_{m}\right\}$ denotes the set of edges referred to all the communication links. If $\left\{v_{i}, v_{j}\right\} \subseteq V$, the edge $e=\left(v_{i}, v_{j}\right) \in E$ if and only if $v_{j}$ is located within the communication range

of $v_{i}$. We assume that nodes have the ability of data aggregation and can use one time slot to transmit data in one link.

Each node operates in three states: active state (transmit, receive and listen), sleep state and transient state (state transition). The transient state comprises two processes: startup (from the sleep state to the active state) and turndown (from the active state to the sleep state). The startup process from the sleep state to the active state includes radio initialization, radio and its oscillator startup, and the switch of radio to active [14]. The startup process is slow due to the feedback loop in the phase-locked loop (PLL) [15], and a typical setting time of the PLL-based frequency synthesizer is on the order of milliseconds.

In wireless networks, the packets transmitted by a node can be received by all the nodes within its communication range. Therefore, interferences may occur among these nodes due to the broadcast nature of the wireless medium. Effective wakeup scheduling should allow every node to start up and transmit or receive its messages without interferences, such as hidden terminal problem [16].

\section{B. Problem Formulation}

In TDMA wakeup schedulings, each bidirectional communication link $l_{i j}$ is assigned two time slots: one time slot is that $v_{i}$ is a transmitter and $v_{j}$ is a receiver, while the other one is that $v_{j}$ 


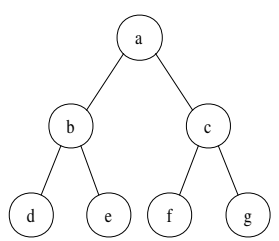

(a)

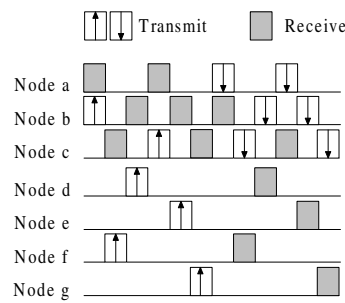

(b)

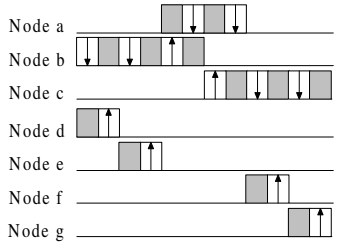

(c)

Fig. 2: Wakeup scheduling and compact wakeup scheduling: (a) network topology, (b) wakeup scheduling, (c) compact wakeup scheduling

is a transmitter and $v_{i}$ is a receiver. In the two time slots, nodes $v_{i}$ and $v_{j}$ start up, and switch from the sleep state to the active state. After that, nodes $v_{i}$ and $v_{j}$ switch to the sleep state again. We can see that node $v_{i}$ may start up $2 w_{i}$ times to communicate bidirectionally with its neighbors in a scheduling period $T$ in the worst case, where $w_{i}$ is the number of neighbors of $v_{i}$. To minimize the frequency of state transitions, we propose a new scheduling approach called compact wakeup scheduling.

Definition 1. Compact wakeup scheduling is an interference-free wakeup scheduling to assign consecutive time slots to all the links incident to a node $v_{i}$, and then $v_{i}$ needs to start up only once to communicate bidirectionally with all its neighbors. Such a scheduling is said to be valid if all the links incident to $v_{i}$ are assigned consecutive time slots.

In the compact wakeup scheduling, the two time slots assigned to each bidirectional link $l_{i j}$ are adjacent, and node $v_{i}$ can finish its bidirectional communication with $v_{j}$ in consecutive time slots. Fig. 2 (a) shows the given network topology. Fig. 2 (b) shows a wakeup scheduling, in which a node starts up numerous times in a period. Fig. 2 (c) shows a compact wakeup scheduling, in which a node could start up only once to communicate bidirectionally with its neighbors. Compact wakeup scheduling can reduce the time for a node to collect the data from its neighbors. As shown in Fig. 2 (b) and (c), node $c$ needs 5 more time slots to communicate with all its neighbors without the compact wakeup scheduling.

An edge coloring of graph $G$ is called a valid coloring if any two adjacent edges of $G$ are assigned different colors. A valid coloring of $G$ is called an interval (or consecutive) edgecoloring if, for each vertex $v$, the colors of edges incident to $v$ form an integer interval. 


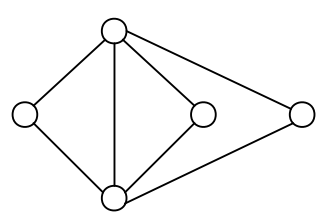

(a) Clas s 1

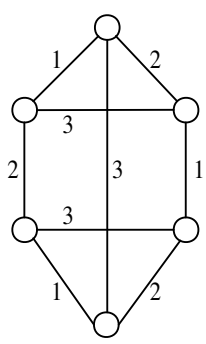

(b) Interval edge-coloring

Fig. 3: Class 1 graphs without valid compact wakeup schedulings

Theorem 1. The problem of deciding whether a valid compact wakeup scheduling exists for an arbitrary graph $G$ is NP-complete.

Proof: The problem is clearly in NP since an assignment can be verified in polynomial time.

To prove that the compact wakeup scheduling problem is NP-hard, we first restate the interval edge-coloring problem with forbidden colors which is NP-complete [17], [18], "Given a graph $G$, a forbidding function $F$ which represents the colors that cannot be assigned to each edge $e$, and an integer $k$, does there exist an interval edge-coloring of $G$ using $k$ colors and avoiding $F$ ?". The interference, such as the hidden terminal problem, in the compact wakeup scheduling is a special case of the forbidding function in the interval edge-coloring. Thus, the compact wakeup scheduling is equivalent to the interval edge-coloring with forbidden colors, which is NP-hard. Therefore, the problem is NP-complete.

Theorem 2. A communication graph $G$ with a valid compact wakeup scheduling has an interval edge-coloring, and belongs to Class 1 graphs.

Proof: If graph $G$ has a valid compact wakeup scheduling, any node $v_{i}$ in $G$ can wake up once to communicate with all its neighbors. Each two-way communication link can be colored with one color, and then the links incident to one node are assigned consecutive colors. Thus, graph $G$ has an interval edge-coloring. According to [18], graph with an interval edge-coloring belongs to Class 1 graphs where the edge chromatic number is equal to the maximum degree $\Delta$ of graph $G$. Therefore, graph $G$ is a Class 1 graph.

Unfortunately, the converse proposition is not true. The graph in Fig. 3 (a) belongs to Class 


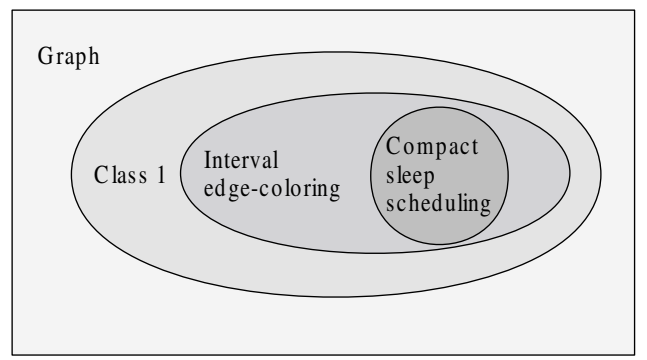

Fig. 4: The relationship among Class 1 graphs, graphs with valid interval edge-colorings and graphs with valid compact wakeup schedulings

1 graphs, but has no valid interval edge-coloring, and thus it has no valid compact wakeup schedulings. The Class 1 graphs even with valid interval edge-colorings may not have valid compact wakeup schedulings. For example, the graph in Fig. 3 (b) has an interval edge-coloring, but all valid interval edge-colorings could not avoid the hidden terminal problem. Thus, graphs with valid compact wakeup schedulings are a proper subset of graphs with valid interval edgecolorings, and also a proper subset of Class 1 graphs, as shown in Fig. 4.

Since not all communication graphs have valid compact wakeup schedulings and the problem of deciding whether a valid scheduling exists for an arbitrary graph is NP-complete, we will focus on particular graphs, such as tree and grid topologies. Interestingly and surprisingly, we can obtain polynomial-time algorithms using the optimum number of time slots in a period. By minimizing the number of time slots, the overall network throughput can be maximized.

\section{Compact Wakeup Scheduling Algorithms}

In this section, we propose polynomial-time algorithms to produce valid compact wakeup schedulings for tree and grid topologies, which are commonly used in WSNs. Note that compact wakeup schedulings also exist in other types of graphs, such as line and hexagonal grid topologies, and the schedulings can be obtained using algorithms similar to that in tree and grid topologies.

\section{A. Trees}

To obtain a valid compact wakeup scheduling of a tree, we first obtain an interval edge-coloring of a tree, then try to assign time slots to each edge and make it interference-free. If graph $G$ is a 
tree of degree $\Delta$, we could get an interval edge-coloring of $G$ using color $1, \cdots, \Delta$. In the coloring process (lines 1 to 9 in Algorithm 1), when coloring a new uncolored edge, the consecutiveness of edge-coloring remains invariant, and the edges already colored form a consecutively colored subgraph. After all edges are colored, we could get an interval edge-coloring and the total number of colors assigned is $\Delta$.

\section{Algorithm 1 Compact wakeup scheduling of a tree}

Input: A tree $G=(V, E)$.

Output: A valid compact wakeup scheduling.

// Interval edge-coloring of a tree

1: Color any edge with 1.

2: while there are uncolored edges do

3: $\quad$ Find a uncolored edge $e$ whose end vertex $v$ is adjacent to an already colored edge. Let $\{a, \cdots, b\}$ be the interval of colors assigned to $v$.

4: $\quad$ if $a>1$ then

5: $\quad$ Color edge $e$ with $a-1$.

6: $\quad$ else

7: $\quad$ Color edge $e$ with $b+1$.

8: $\quad$ end if

9: end while

// Time slot assignment

10: Map each color to two consecutive time slots, and use Algorithm 2 to determine a valid direction of transmission assignment to avoid interferences.

We now describe how the interval edge-coloring is used to assign time slots to each edge. The idea is to map each color to two consecutive time slots and assign a valid direction of transmission to avoid interferences. In Fig. 5, link $l_{a b}$ and $l_{c e}$ are assigned the same color "1" in the interval edge-coloring, while time slot $t s_{1}$ and $t s_{2}$ are allocated for color "1". If time slot $t s_{1}$ is assigned in the directions of transmission as shown in Fig. 5 (a), the hidden terminal problem would happen because the reception at node $v_{b}$ is garbled due to the collision of transmission from nodes $v_{a}$ and $v_{c}$. Alternatively, if time slot $t s_{1}$ is assigned in the directions of transmission 


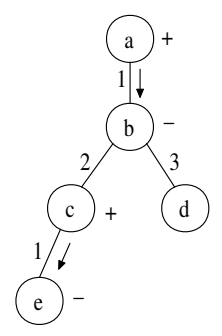

(a)

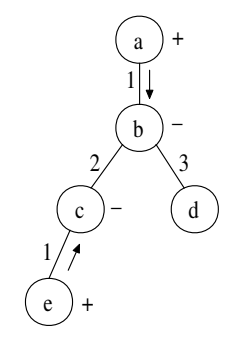

(b)

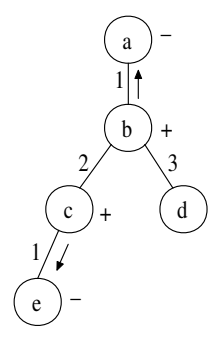

(c)

Fig. 5: Compact wakeup scheduling of a tree: (a) hidden terminal problem, (b) avoid the hidden terminal problem, (c) avoid the exposed terminal problem

as shown in Fig. 5 (b), the hidden terminal problem could be avoided. Similarly, time slot $t s_{2}$ is assigned in the reverse directions of transmission as shown in Fig. 5 (c). Inspired by this, we should determine the directions of transmission along each link carefully to avoid the hidden terminal problem, i.e., determine a node when to transmit and when to receive.

We first introduce how to avoid the hidden terminal problem after a valid coloring is obtained. In this paper, the transmitter is marked with a sign "+" and the receiver is marked with a sign “-”. Given a coloring of graph $G$ and a color $k$, a subgraph $G^{k}=\left(V^{k}, E^{k}\right)$ is defined as follows: a) $V^{k}$ is the set of vertices incident to the edges colored with $k$. b) $E^{k}$ is the set of edges with both end vertices in $V^{k}$. When a node is assigned a sign "-", the only neighbor assigned a sign "+" in $G^{k}$ is the neighbor incident to the edge colored with $k$, and the other neighbors in $G^{k}$ are individually assigned a sign "-". Then, nodes incident to an edge colored with $k$ always have an opposite sign, and nodes incident to an edge colored with other colors have the same sign. Algorithm 2, based on Depth First Search (DFS), can provide a valid direction of transmission assignment to $G^{k}$. Note that the time slot assignment also avoids the exposed terminal problem [16], as shown in Fig. 5 (c).

Definition 2. The span of a valid compact wakeup scheduling of graph $G$ is the number of colors assigned. The minimum and maximum span over all valid compact wakeup schedulings of $G$ are denoted by $\chi_{c w}(G)$ and $\zeta_{c w}(G)$, respectively.

As any valid coloring in a tree requires at least $\Delta$ colors and an interval edge-coloring can be obtained using $\Delta$ colors, $\chi_{c w}(G)$ is equal to $\Delta$. Then, the number of time slots assigned in 


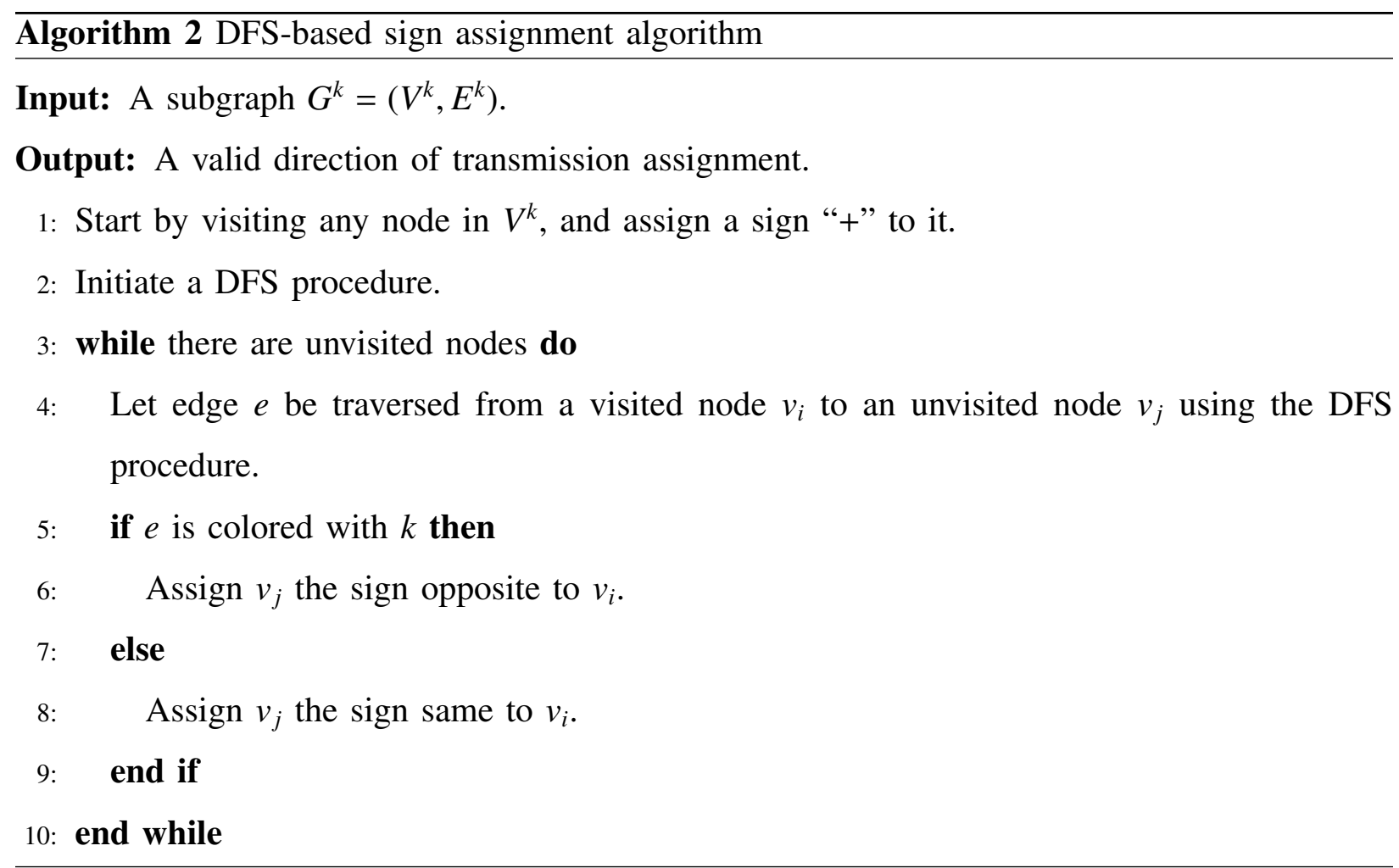

the compact wakeup scheduling is $2 \Delta$, which is the optimum number of time slots. Algorithm 1 describes the compact wakeup scheduling for trees. Both the interval edge-coloring of a tree and the time slot assignments can be obtained using $O(n)$, where $n$ is the number of vertices in a tree. Thus, the algorithm to produce a valid compact wakeup scheduling for trees is polynomial-time.

\section{B. Grid Graphs}

A $\mathcal{V} \times \mathcal{H}$ grid graph $(3 \leq \mathcal{V} \leq \mathcal{H})$ is a square lattice graph composed of $\mathcal{V H}$ vertices. The grid graph has $\mathcal{H}$ vertical paths and $\mathcal{V}$ horizontal paths, where each vertical path consists of $\mathcal{V}$ vertices and each horizontal path consists of $\mathcal{H}$ vertices.

Definition 3. In a $\mathcal{V} \times \mathcal{H}$ grid graph, $\bar{V}_{i j}(1 \leq i \leq \mathcal{V}-1,1 \leq j \leq \mathcal{H})$ denotes the $i^{\text {th }}$ vertical edge in the $j^{\text {th }}$ vertical path, and $\bar{H}_{i j}(1 \leq i \leq \mathcal{V}, 1 \leq j \leq \mathcal{H}-1)$ denotes the $j^{\text {th }}$ horizontal edge in the $i^{\text {th }}$ horizontal path.

Sample grids with labeled vertical and horizontal edges are illustrated in Fig. 6 (a) and (b). $\bar{V}_{i j}$ is called parallel to $\bar{V}_{m n}$ if $i=m, \bar{H}_{i j}$ is called parallel to $\bar{H}_{m n}$ if $j=n$. For example, $\bar{H}_{11}$, 


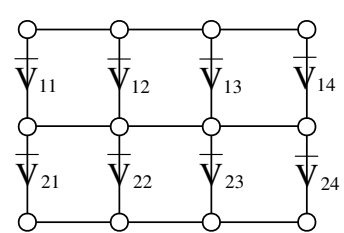

(a) Vertical edges

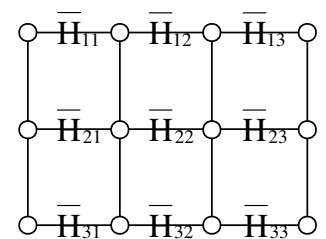

(b) Horizontal edges

Fig. 6: Vertical and horizontal edges in grid graphs

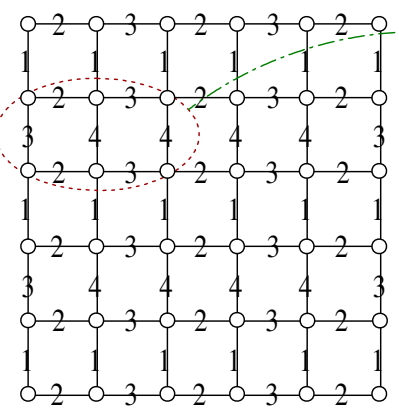

(a)

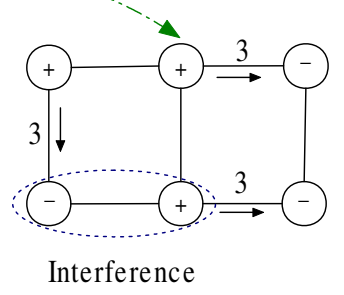

(b)

Fig. 7: An interval edge-coloring of a grid graph: (a) interval edge-coloring, (b) hidden terminal problem

$\bar{H}_{21}$ and $\bar{H}_{31}$ are parallel in Fig. 6 (b).

Grid graphs can be consecutively colored with $\Delta$ colors, and one interval edge-coloring approach is given below: For a $\mathcal{V} \times \mathcal{H}$ grid graph, let $c$ be a consecutive coloring of each horizontal path with colors 2 and 3 . For each $i=1,2, \cdots, \mathcal{V}$, we color the edges of $i^{\text {th }}$ horizontal path according to $c$. Let $\{a, \cdots, b\}$ be an interval of colors assigned at each vertex in the corresponding horizontal path, then edge $\bar{V}_{1 j}$ is colored with $a-1, \bar{V}_{2 j}$ with $b+1, \bar{V}_{3 j}$ with $a-1$, and so forth, where $1 \leq j \leq \mathcal{H}$. By repeating this for all edges, we could obtain a interval edge-coloring of $G$, and a sample of the edge-coloring is shown in Fig. 7 (a).

A valid direction of transmission assignment can be obtained to avoid the hidden terminal problem using Algorithm 2 in acyclic subgraphs $G^{k}$. But grid graphs contain cycles, a valid assignment does not exist if we use the interval edge-coloring approach above. For example, this edge-coloring cannot avoid the hidden terminal problem as shown in Fig. 7 (b). Interestingly, Gandham et al. [12] prove that all the nodes in a cycle of $G^{k}$ can be given a valid sign "+" or 
"-" if and only if there are an even number of edges with color $k$ in the cycle.

If the edges colored with "3" in the cycle of Fig. 7 (b) are assigned with other colors, the consecutiveness of the colors assigned to the edges incident to one node cannot be held. Our solution for a grid graph first considers the property of the hidden terminal problem in the grid graph, and then deals with the consecutiveness of the edge-coloring. Our key results for grid graphs are summarized below:

(1) We obtain an interval edge-coloring according to the parity of $\mathcal{V}$ and $\mathcal{H}$.

* If both $\mathcal{V}$ and $\mathcal{H}$ are even, $\chi_{c w}(G)=4$.

* If one of $\mathcal{V}$ and $\mathcal{H}$ is even and the other is odd, $\chi_{c w}(G)=5$.

* If both $\mathcal{V}$ and $\mathcal{H}$ are odd, $\chi_{c w}(G)=6$.

(2) We point out all the possible coloring patterns.

(3) We give the upper bound of the compact wakeup scheduling.

Definition 4. In a grid graph, the maximum degree of vertices is $\Delta=4$. The vertices of degree 4 are inner vertices, the vertices of degree 2 or 3 are boundary vertices, the edges incident to at least one inner vertex are inner edges, and the edges incident to two boundary vertices are boundary edges.

Definition 5. In the compact wakeup scheduling of a grid graph, the colors assigned to the inner edges incident to an inner vertex form an interval of 4 integers. When the total number of colors assigned is less than 8 , certain color must appear in one of the inner edges and this color is referred to as a critical color.

In grid graphs, if the total number of colors assigned is $M(4 \leq M \leq 7)$, then the number of critical colors is $8-M$. For example, if $M=4$, the set of critical colors is $\{1,2,3,4\}$; if $M=5$, the set of critical colors is $\{2,3,4\}$; if $M=6$, the set of critical colors is $\{3,4\}$.

Lemma 1. If $K$ is a critical color assigned to an inner edge e incident to two inner vertices in the compact wakeup scheduling of a grid graph, the inner edges parallel to e are all colored with $K$.

Proof: Without loss of generality, we assume that an inner horizontal edge $\bar{H}_{i j}(2 \leq i \leq$ $\mathcal{V}-1,2 \leq j \leq \mathcal{H}-2$ ) is colored with $K$ in a $\mathcal{V} \times \mathcal{H}$ grid graph. The cases of colorings shown 


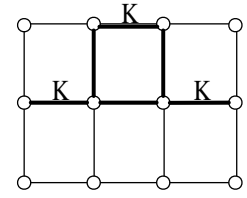

(a)

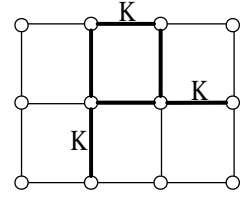

(b)

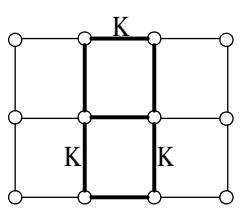

(c)

Fig. 8: Invalid colorings in the compact wakeup scheduling

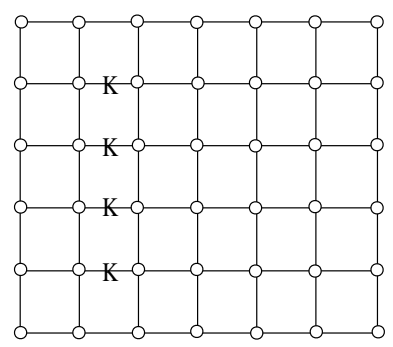

(a) Lemma 1

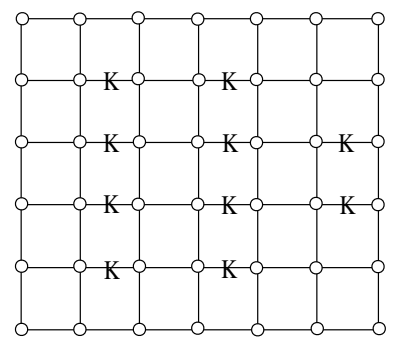

(b) Lemma2

Fig. 9: Coloring pattern in the compact wakeup scheduling

in Fig. 8 would lead to odd number of edges with color $K$ in subgraph $G^{K}$ (see the thick lines in Fig. 8), and no feasible direction of transmission can be obtained. Since $K$ is a critical color, $\bar{H}_{(i+1) j}(i+1 \leq \mathcal{V}-1)$ must be colored with $K$. By applying recursion, the horizontal edges $\bar{H}_{m j}(2 \leq m \leq \mathcal{V}-1)$ are in a parallel pattern, as shown in Fig. 9 (a).

Lemma 2. If $K$ is a critical color, the inner edges colored with $K$ are in an interlined pattern.

Proof: Without loss of generality, we assume an inner horizontal edge $\bar{H}_{i j}(2 \leq i \leq \mathcal{V}-1,2 \leq$ $j \leq \mathcal{H}-2$ ) is colored with $K$ in a $\mathcal{V} \times \mathcal{H}$ grid graph. According to Lemma 1 , the horizontal edges $\bar{H}_{m j}(2 \leq m \leq \mathcal{V}-1)$ are colored with $K$. Let $k=j+2(k \leq \mathcal{H}-2), \bar{H}_{3 k}$ must be colored with $K$, since $K$ is a critical color and $\bar{H}_{3(k-1)}, \bar{V}_{2 k}$ as well as $\bar{V}_{3 k}$ cannot be colored with $K$. According to Lemma 1 , the horizontal edges $\bar{H}_{m k}(2 \leq m \leq \mathcal{V}-1)$ are colored with $K$, and the result still holds when $k=j-2(k \geq 2)$. By applying recursion, the horizontal edges $\bar{H}_{m k}(k=j \pm 2 n, n \in N, 2 \leq m \leq \mathcal{V}-1,2 \leq k \leq \mathcal{H}-2)$ are colored with $K$. If $k=1$ (or $\left.\mathcal{H}-1\right)$ and $k=j \pm 2 n, n \in N$, the horizontal edges $\bar{H}_{m k}(3 \leq m \leq \mathcal{V}-2)$ are colored with $K$, since $K$ is a critical color. Hence, the inner edges colored with a critical color are in an interlined pattern, as shown in Fig. 9 (b). 


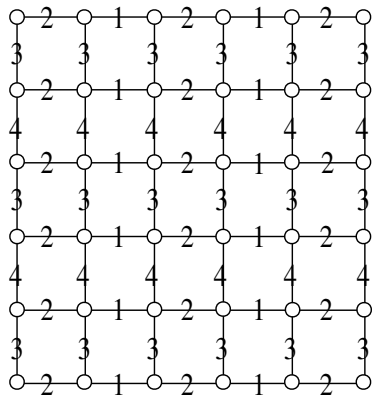

(a) evenxeven (pattern 1)

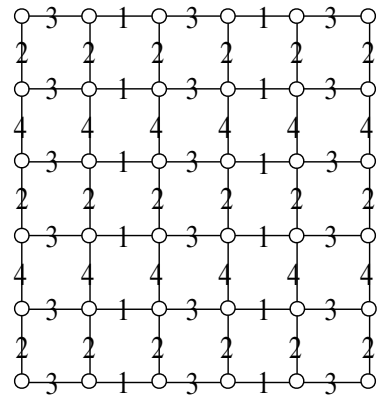

(b) evenxeven (pattern 2)

Fig. 10: The coloring patterns in the compact wakeup scheduling (Both $\mathcal{V}$ and $\mathcal{H}$ are even.)

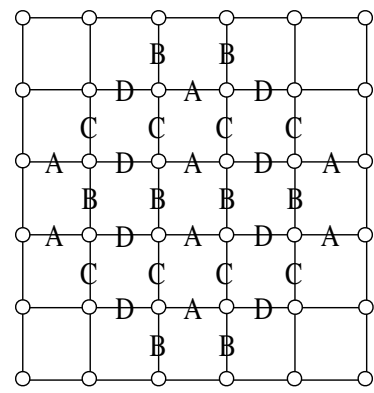

(a) evenxeven (status 1)

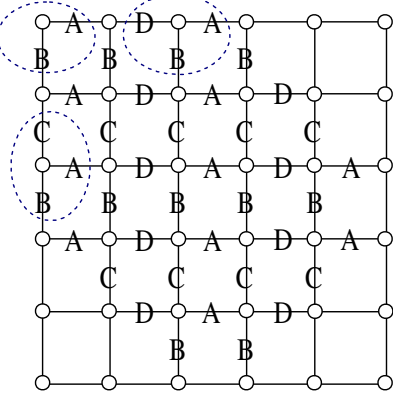

(b) evenxeven (status 2)

Fig. 11: The coloring in the compact wakeup scheduling (Both $\mathcal{V}$ and $\mathcal{H}$ are even.)

Theorem 3. $A \mathcal{V} \times \mathcal{H}$ grid graph $(3 \leq \mathcal{V} \leq \mathcal{H}$, both $\mathcal{V}$ and $\mathcal{H}$ are even) can be consecutively colored with 4 colors in the compact wakeup scheduling, and the possible colorings must be the patterns as shown in Fig. 10, and $\chi_{c w}(G)=4$.

Proof: Fig. 10 (a) and (b) show two possible colorings in the compact wakeup scheduling, if both $\mathcal{V}$ and $\mathcal{H}$ are even. Since the edges with the same color are in a parallel and interlined pattern, there are an even number of edges with color $k(1 \leq k \leq 4)$ in a cycle in the subgraph $G^{k}$ and then the scheduling could avoid the hidden terminal problem. Therefore, $\chi_{c w}(G)=4$.

As $\chi_{c w}(G)$ is equal to 4 , we assume the four critical colors are $A, B, C$ and $D$. According to Lemma 1 and Lemma 2, $A, B, C$ and $D$ are all in a parallel and interlined pattern shown as the status 1 in Fig. 11 (a). To avoid the hidden terminal problem, $\bar{H}_{13}$ cannot be colored with $D$ or $C$, and can only be colored with $A$. Then $\bar{H}_{12}$ must be colored with $D$. Similarly, $\bar{V}_{21}$ and $\bar{V}_{31}$ 


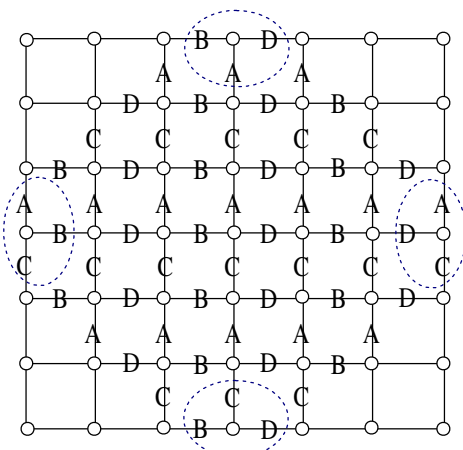

(a) 4 colors

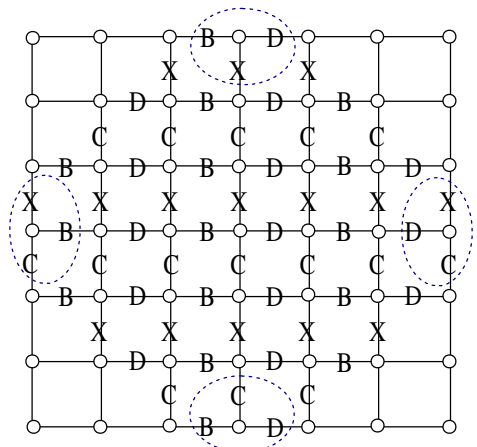

(b) 5 colors

Fig. 12: The colorings in the compact wakeup scheduling using 4 and 5 colors (Both $\mathcal{V}$ and $\mathcal{H}$ are odd.)

are colored with $C$ and $B$, respectively. Then $\bar{H}_{11}, \bar{V}_{11}, \bar{H}_{21}$ and $\bar{V}_{12}$ are colored with $A, B, A$ and $B$, respectively. We can color other edges in a similar way. In the status 2 shown in Fig. 11 (b), we can see the color sets $\{A, B\},\{A, B, D\}$ and $\{A, B, C\}$ must consist of consecutive numbers since these colors assigned to the edges incident to the vertices in the dashed circles must be consecutive. Therefore, $\{A, B, C\}$ and $\{A, B, D\}$ belong to $\{1,2,3\}$ and $\{2,3,4\},\{A, B\}$ belongs to $\{2,3\}$. For $C$ and $D$ are symmetrical, we can get $C=4$ and $D=1$. For the case that $A=2$ and $B=3$, the coloring pattern is Fig. 10 (a). For the case that $A=3$ and $B=2$, the coloring pattern is Fig. $10(\mathrm{~b})$.

Lemma 3. $A \mathcal{V} \times \mathcal{H}$ grid graph $(3 \leq \mathcal{V} \leq \mathcal{H}$, both $\mathcal{V}$ and $\mathcal{H}$ are odd $)$ cannot be consecutively colored with 4 or 5 colors in the compact wakeup scheduling.

Proof: 1) If the grid could be consecutively colored with 4 colors $A, B, C$ and $D$, the four colors belonging to $\{1,2,3,4\}$ are all critical colors. For an inner vertex has 4 incident inner edges, the inner edges are colored with $A, B, C$ and $D$, respectively. According to Lemma 1 and Lemma 2, $A, B, C$ and $D$ are all in a parallel and interlined pattern, and the coloring is shown in Fig. 12 (a). As the colors assigned to the edges incident to the vertices in the dashed circles must be consecutive, the color sets $\{A, B, C\},\{B, C, D\},\{A, C, D\}$ and $\{A, B, D\}$ must consist of three consecutive numbers. However, $\{1,2,3\}$ and $\{2,3,4\}$ are the only two possible cases with three consecutive numbers, which leads to a contradiction.

2) If the grid could be consecutively colored with 5 colors, $B, C$ and $D$ belonging to $\{2,3,4\}$ are 


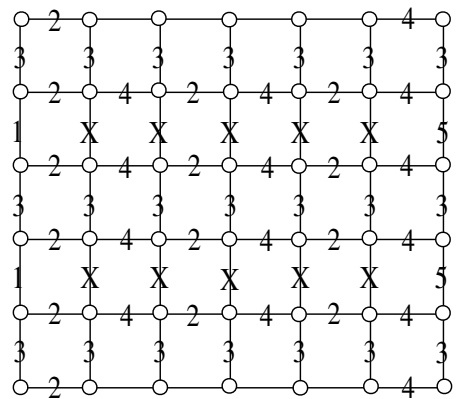

(a) evenxodd (general pattern)

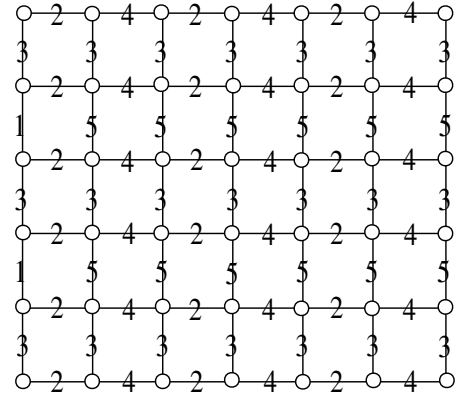

(b) even $\times$ odd (pattern)

Fig. 13: The general coloring pattern and coloring pattern in the compact wakeup scheduling (One of $\mathcal{V}$ and $\mathcal{H}$ is even and the other is odd. The uncolored edges depend on the edges colored with $X$, and $X=1$ or 5 .)

critical colors and the non-critical color 1 or 5 is denoted by $X$. For an inner vertex has 3 incident critical inner edges, the inner edges are colored with $B, C$ and $D$, respectively. According to Lemma 1 and Lemma 2, B, $C$ and $D$ are all in a parallel and interlined pattern, and the coloring is shown in Fig. 12 (b). Since the colors assigned to the edges incident to the vertices in the dashed circles must be consecutive, the color sets $\{B, C, X\},\{B, C, D\},\{C, D, X\}$ and $\{B, D, X\}$ must consist of three consecutive numbers. However, $\{1,2,3\},\{2,3,4\}$ and $\{3,4,5\}$ are the only three possible cases with three consecutive numbers, which leads to a contradiction.

Similarly, we could get the following lemma.

Lemma 4. $A \mathcal{V} \times \mathcal{H}$ grid graph $(3 \leq \mathcal{V} \leq \mathcal{H}$, one of $\mathcal{V}$ and $\mathcal{H}$ is even and the other is odd) cannot be consecutively colored with 4 colors in the compact wakeup scheduling.

Theorem 4. $A \mathcal{V} \times \mathcal{H}$ grid graph $(3 \leq \mathcal{V} \leq \mathcal{H}$, one of $\mathcal{V}$ and $\mathcal{H}$ is even and the other is odd $)$ can be consecutively colored with 5 colors in the compact wakeup scheduling, and the possible coloring must be the pattern as shown in Fig. $13(a)$, and $\chi_{c w}(G)=5$.

Proof: Fig. 13 (b) shows a possible coloring in the compact wakeup scheduling by determining the colors for the rest uncolored edges in Fig. 13 (a), if one of $\mathcal{V}$ and $\mathcal{H}$ is even and the other is odd. Since the edges with the same color are in a parallel and interlined pattern, there are an even number of edges with color $k(1 \leq k \leq 5)$ in a cycle in the subgraph $G^{k}$ and then the 


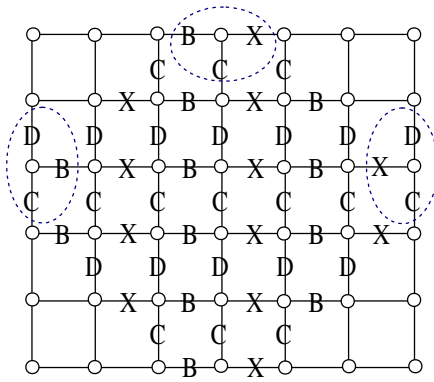

(a) even $\times$ odd (case 1, status 1)

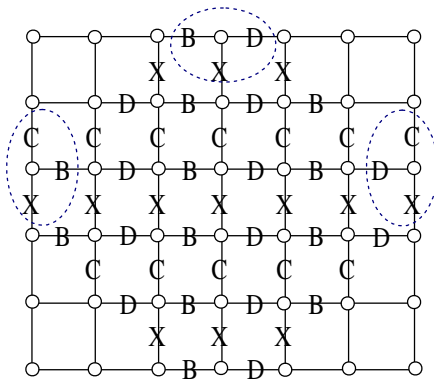

(c) evenxodd (case 2)

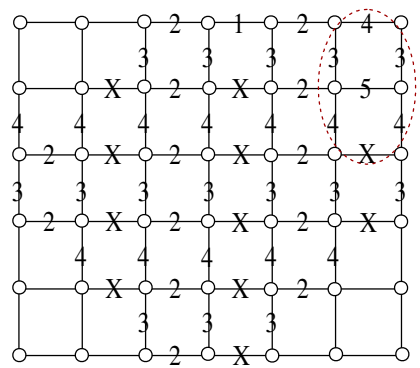

(b) evenxodd (case 1, status 2)

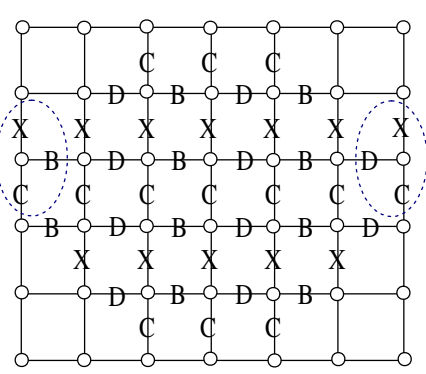

(d) evenxodd (case 3)

Fig. 14: The colorings in the compact wakeup scheduling (One of $\mathcal{V}$ and $\mathcal{H}$ is even and the other is odd.)

scheduling could avoid the hidden terminal problem. By combining with Lemma 4, $\chi_{c w}(G)=5$.

Since $\chi_{c w}(G)$ is equal to 5 , we assume $B, C$ and $D$ belonging to $\{2,3,4\}$ are critical colors and the non-critical color 1 or 5 is denoted by $X$. As an inner vertex has 3 incident critical inner edges, Fig. 14 (a), (c) and (d) are the possible coloring patterns.

Case 1: In the status 1 shown in Fig. 14 (a), $\bar{H}_{14}$ cannot be colored with $B, C$ or $D$, and can only be colored with $X$. Then $\bar{H}_{13}$ must be colored with $B$. Similarly, $\bar{V}_{21}, \bar{V}_{31}, \bar{V}_{27}, \bar{V}_{37}$ are colored with $D, C, D$ and $C$, respectively. We can see that the color sets $\{B, C, X\},\{B, C, D\}$ and $\{C, D, X\}$ must consist of consecutive numbers since these colors assigned to the edges incident to the vertices in the dashed circles must be consecutive. Then, $\{B, C, X\}$ and $\{C, D, X\}$ belong to $\{1,2,3\}$ and $\{3,4,5\}$. Then, we can get $C=3$. If $B=2$ and $D=4, \bar{H}_{15}, \bar{V}_{16}, \bar{H}_{26}$ and $\bar{V}_{17}$ are colored with 2, 3, 5 and 3, respectively, shown as the status 2 in Fig. 14 (b). Then $\bar{H}_{16}$ can only be colored with 4 , which leads to interferences in the dashed circle. Similarly, if $B=4$ and $D=2$, we cannot get an interference-free scheduling either. Hence, the coloring pattern in Fig. 14 (a) is not valid. 
Case 2: In Fig. 14 (c), $\bar{H}_{14}$ cannot be colored with $B, C$ or $X$, and can only be colored with $D$. Then $\bar{H}_{13}$ must be colored with $B$. Similarly, $\bar{V}_{21}, \bar{V}_{31}, \bar{V}_{27}, \bar{V}_{37}$ are colored with $C$, $X, C$ and $X$, respectively. We can see that the color sets $\{B, D, X\},\{B, C, X\}$ and $\{C, D, X\}$ must consist of consecutive numbers since these colors assigned to the edges incident to the vertices in the dashed circles must be consecutive. Moreover, $B, C, D \in\{2,3,4\}$ are consecutive. However, $\{1,2,3\},\{2,3,4\}$ and $\{3,4,5\}$ are the only three possible cases with three consecutive numbers. Hence, the coloring pattern in Fig. 14 (c) is not valid.

Case 3: In Fig. 14 (d), $\bar{V}_{21}$ cannot be colored with $B, C$ or $D$, and can only be colored with $X$. Then $\bar{V}_{31}$ must be colored with $C$. Similarly, $\bar{V}_{27}$ and $\bar{V}_{37}$ are colored with $X$ and $C$, respectively. We can see that the color sets $\{B, C, X\}$ and $\{C, D, X\}$ must consist of consecutive numbers since these colors assigned to the edges incident to the vertices in the dashed circles must be consecutive. Then $C=3$. For $B$ and $D$ are symmetrical, we can get $B=2$ and $D=4$. By assigning the possible colors in other edges, the coloring pattern in Fig. 13 (a) is obtained.

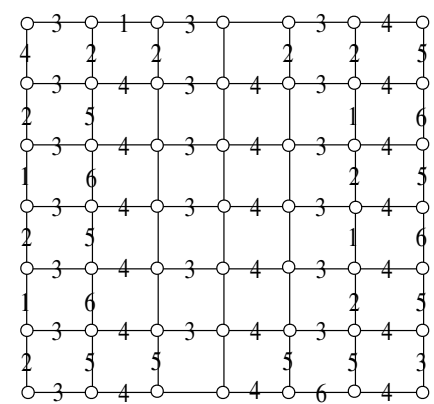

(a) odd $\times$ odd (general pattern 1)

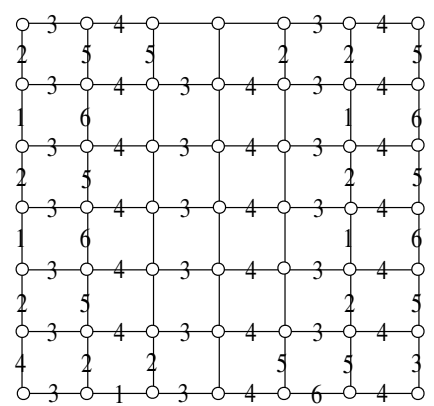

(b) odd xodd (general pattern 2)

Fig. 15: The general coloring patterns in the compact wakeup scheduling (Both $\mathcal{V}$ and $\mathcal{H}$ are odd. The uncolored edges have various alternatives. )

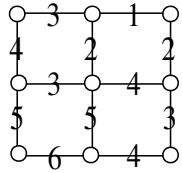

(a) $3 \times 3$

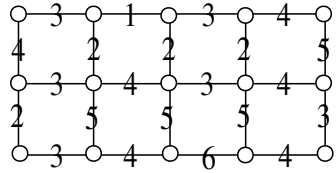

(b) $3 \times 5$

Fig. 16: The coloring patterns in the compact wakeup scheduling ( $\mathcal{V}=3$ and $\mathcal{H}$ is odd.) 


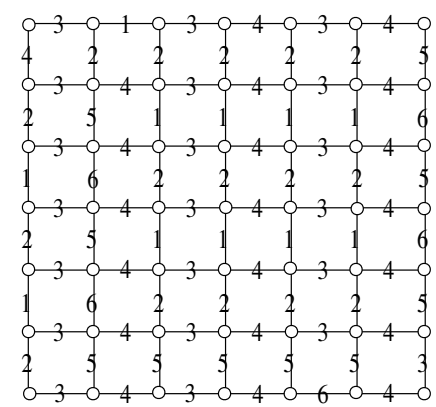

(a) odd xodd (pattern 1)

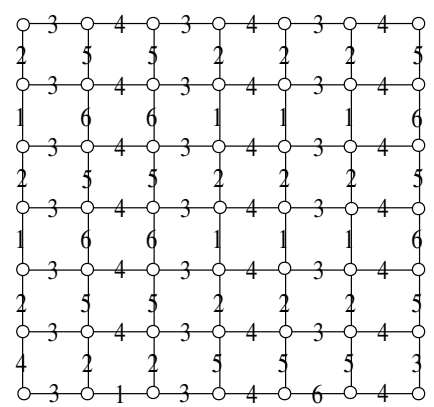

(b) oddxodd (pattern 2)

Fig. 17: The coloring patterns in the compact wakeup scheduling (Both $\mathcal{V}$ and $\mathcal{H}$ are odd.)

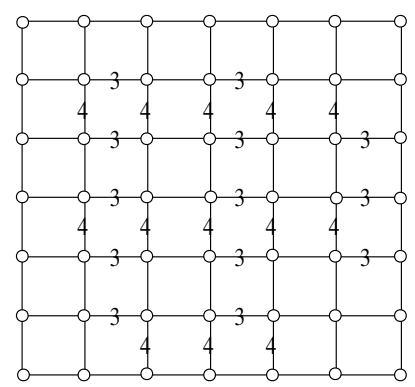

(a) odd xodd (case 1)

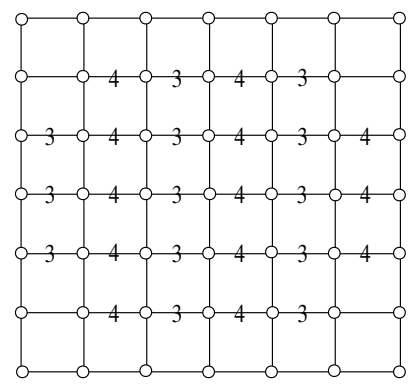

(c) odd xodd (case 2)

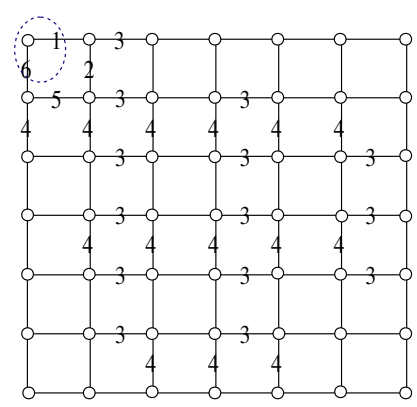

(b) odd xodd (case 1)

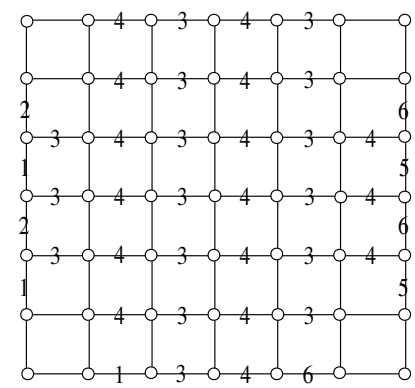

(d) odd xodd (case 2)

Fig. 18: The colorings in the compact sleep scheduling (both $\mathcal{V}$ and $\mathcal{H}$ are odd)

Theorem 5. A $\mathcal{V} \times \mathcal{H}$ grid graph $(3 \leq \mathcal{V} \leq \mathcal{H}$, both $\mathcal{V}$ and $\mathcal{H}$ are odd) can be consecutively colored with 6 colors in the compact wakeup scheduling, and the possible colorings must be the patterns as shown in Fig. 15, and $\chi_{c w}(G)=6$.

Proof: Fig. 17 (a) and (b) shows two possible colorings in the compact sleep scheduling by determining the colors for the rest uncolored edges in Fig. 15 (a) and (b), if both $\mathcal{V}$ and $\mathcal{H}$ 
are odd. Specially, if $\mathcal{V}=3$, the possible colorings are shown in Fig. 16 (a) and (b). Since there are even number of edges with color $k(1 \leq k \leq 6)$ in a circle in the subgraph $G^{k}$ and then the scheduling could avoid the hidden terminal problem. According to Lemma 3, $\chi^{\prime}(G)=6$.

Since the grid graph can be consecutively colored with 6 colors, 3 and 4 are critical colors. For an inner vertex has two incident critical inner edges, Fig. 18 (a) and (c) are the possible coloring patterns.

Case 1: In Fig. 18 (a), $\bar{V}_{21}, \bar{V}_{31}$ and $\bar{H}_{31}$ cannot be colored with 3, can only be colored with $\{4,5,6\}$. For $\bar{V}_{31}$ and $\bar{H}_{31}$ cannot be colored with $4, \bar{V}_{21}$ must be colored with 4 . Similarly, $\bar{H}_{12}$ must be colored with 3. Then $\bar{V}_{11}, \bar{V}_{21}$ and $\bar{H}_{21}$ must be colored with $\{4,5,6\}$, and $\bar{H}_{11}, \bar{H}_{12}$ and $\bar{V}_{12}$ must be colored with $\{1,2,3\}$. For $\bar{V}_{12}, \bar{V}_{22}, \bar{H}_{21}$ and $\bar{H}_{22}$ are consecutively colored, $\bar{V}_{12}$ is colored with 2 and $\bar{H}_{21}$ is colored with 5. Then, $\bar{V}_{11}$ is colored with 6 and $\bar{H}_{11}$ is colored with 1, which leads to an inconsecutive coloring, as shown in Fig. 18 (b). Hence, Fig. 18 (a) is not a possible coloring.

Case 2: In Fig. 18 (c), $\bar{V}_{21}, \bar{V}_{23}$ and $\bar{H}_{31}$ cannot be colored with 4, can only be colored with $\{1,2,3\} ; \bar{V}_{27}, \bar{V}_{37}$ and $\bar{H}_{36}$ cannot be colored with 3 , can only be $\{4,5,6\}$. According to the symmetrical property, we suppose $\bar{V}_{27}$ and $\bar{V}_{37}$ are colored with 6 and 5, respectively. If $\bar{V}_{21}$ is colored with 2 and $\bar{V}_{31}$ is colored with 1, we can get Fig. 18 (d). By assigning the possible colors in other edges, the coloring pattern in Fig. 15 (a) is obtained. If $\bar{V}_{21}$ is colored with 1 and $\bar{V}_{31}$ is colored with 2, we can get the coloring pattern in Fig. 15 (b).

Hence, the possible colorings must be the patterns as shown in Fig. 15 (a) and (b), and $\chi^{\prime}(G)=6$.

Theorem 6. In the compact wakeup scheduling of a $\mathcal{V} \times \mathcal{H}$ grid graph $(3 \leq \mathcal{V} \leq \mathcal{H})$, $2 \mathcal{V}+$ $2 \mathcal{H}-6 \leq \zeta_{c w}(G) \leq \frac{1}{6}(13 \mathcal{V}+13 \mathcal{H}-8)$.

Proof: Lower bound: We can get a valid consecutive edge coloring with a valid direction of transmission assignment in the compact wakeup scheduling using $2 \mathcal{V}+2 \mathcal{H}-6$ colors. For example, the number of colors assigned is $22=2 \times 7+2 \times 7-6$ in a $7 \times 7$ grid as shown in Fig. 19 (a).

Upper bound: For a consecutive edge coloring in the compact wakeup scheduling of a grid graph $G$, the difference in colors of edges incident to a node $v$ cannot exceed $\operatorname{deg}\left(v_{i}\right)-1$. Suppose that $v_{1}, v_{2}, \cdots, v_{m}$ is the vertex sequence of a path connecting edges with extremal colors, we 


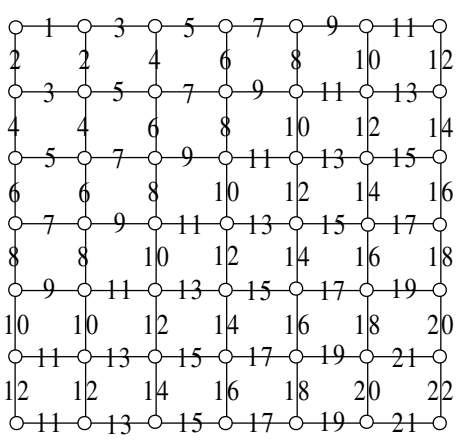

(a)

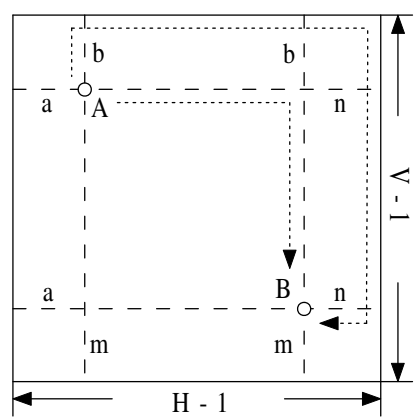

(b)

Fig. 19: $\zeta_{c w}(G)$ in the compact wakeup scheduling: (a) lower bound of $\zeta_{c w}(G)$, (b) upper bound of $\zeta_{c w}(G)$

could get $\zeta_{c w}(G) \leq 1+\sum_{i=1}^{m}\left(\operatorname{deg}\left(v_{i}\right)-1\right)$. We suppose vertices $A$ and $B$ are on the path connecting edges with minimum and maximum colors, respectively, as shown in Fig. 19 (b). We assume vertex $A$ is on the common point of $\bar{H}_{(b+1)(a+1)}$ and $\bar{V}_{(b+1)(a+1)}$, and vertex $B$ is on the common point of $\bar{H}_{(\mathcal{V}-m)(\mathcal{H}-1-n)}$ and $\bar{V}_{(\mathcal{V}-1-m)(\mathcal{H}-n)}$. We can get $\zeta_{c w}(G) \leq 1+3(\mathcal{H}-1-a-n+1)+3(\mathcal{V}-1-b-m)=$ $3(\mathcal{V}+\mathcal{H}-a-b-m-n)-2$ using route 1 . We have also known $\zeta_{c w}(G) \geq 2 \mathcal{V}+2 \mathcal{H}-6$ $3(\mathcal{V}+\mathcal{H}-a-b-m-n)-2$ should be no less than $2 \mathcal{V}+2 \mathcal{H}-6$. Otherwise, $2 \mathcal{V}+2 \mathcal{H}-6$ should also be the upper bound. Then we get, $\mathcal{V}+\mathcal{H}+4 \geq 3(a+b+m+n)$. Without loss of generality, we assume $a+m \geq b+n$. Then, $b+n \leq \frac{1}{6}(\mathcal{V}+\mathcal{H}+4)$. We can also get $\zeta_{c w}(G) \leq 1+3 b+2(\mathcal{H}-a-1)+1+2(\mathcal{V}-m-1)+3 n=2(\mathcal{V}+\mathcal{H}-a-m)+3 b+3 n-2$ using route 2. Then, $\zeta_{c w}(G)=2(\mathcal{V}+\mathcal{H})+3(b+n)-2(a+m)-2 \leq 2(\mathcal{V}+\mathcal{H})+b+n-2 \leq \frac{1}{6}(13 \mathcal{V}+13 \mathcal{H}-8)$.

Thus, $\zeta_{c w}(G)$ is bounded by $2 \mathcal{V}+2 \mathcal{H}-6$ and $\frac{1}{6}(13 \mathcal{V}+13 \mathcal{H}-8)$.

According to Theorem 3, 4 and 5, the number of time slots assigned is optimum. If both $\mathcal{V}$ and $\mathcal{H}$ are even, the number of time slots assigned in a period is $4 \times 2=8$ in a $\mathcal{V} \times \mathcal{H}$ grid graph. If one of $\mathcal{V}$ and $\mathcal{H}$ is even and the other is odd, the number of time slots is $5 \times 2=10$. If both $\mathcal{V}$ and $\mathcal{H}$ are odd, the number of time slots is $6 \times 2=12$. Algorithm 3 describes the compact wakeup scheduling of a grid graph and its complexity is $O(n)$.

\section{COMpact Wakeup Deficiency of a GRaph}

Since not all graphs have valid compact wakeup schedulings, one approach is to define a measure of insusceptibility to the problem. We define the compact wakeup deficiency of a graph 


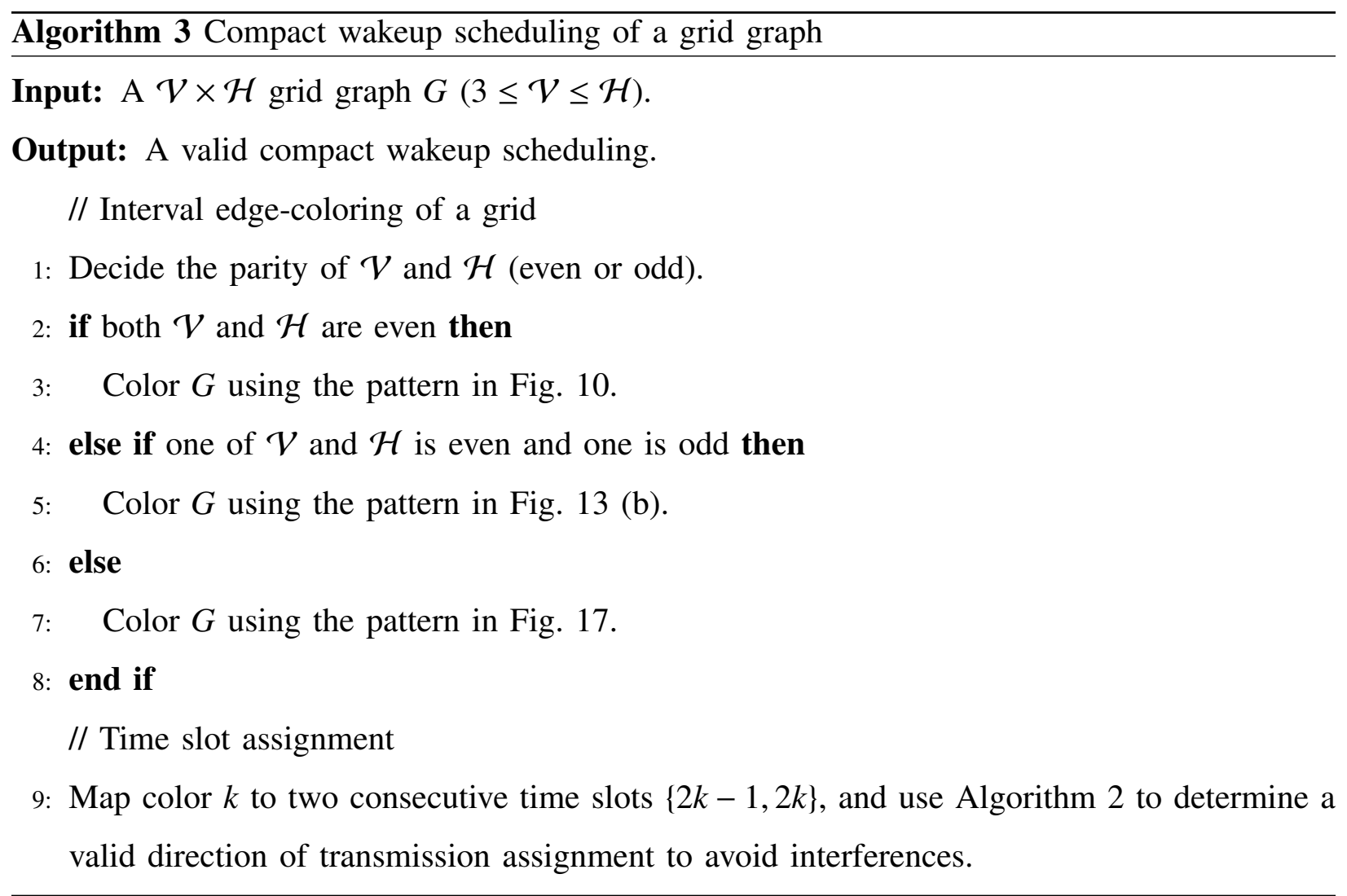

in the case of graphs without valid compact wakeup schedulings.

Definition 6. Given a finite integer set $A$, deficiency $d(A)$ is the smallest number of sets $B_{i}$ such that $A \cup B_{1} \cup B_{2} \cup \cdots \cup B_{k}$ forms a set of consecutive integers and $A \cap B_{i}=\emptyset(1 \leq i \leq k)$, i.e., $d(A)=k$, where $B_{i}$ is a set of consecutive integers.

Definition 7. Given a graph $G=(V, E)$ and an edge-coloring $c$ in compact sleep scheduling, $d(G, c, v)$ denotes the compact wakeup deficiency of vertex $v$ in an edge-coloring $c$ of $G$, which is the deficiency of the set of colors assigned to the edges incident to $v ; d(G, c)$ denotes the compact wakeup deficiency of coloring $c$ of $G$, which is the sum of compact wakeup deficiencies of all vertices, i.e., $d(G, c)=\sum_{v \in V} d(G, c, v) ; d(G)$ denotes the compact wakeup deficiency of graph $G$, which is the minimum $d(G, c)$ of all possible edge-colorings $c$. Graph $G$ is called $d(G)$-deficient. Theorem 7. Given a graph $G$ and a positive integer $k$, the problem of deciding whether $d(G) \leq k$ is NP-complete. 
Proof: According to the definition of the compact wakeup deficiency, graphs with valid compact wakeup schedulings are 0 -deficient. By combining with Theorem 1, the problem of deciding whether $G$ is 0 -deficient is NP-complete. Therefore, the problem of deciding whether the compact wakeup deficiency of $G$ is not greater than $k$ is NP-complete.

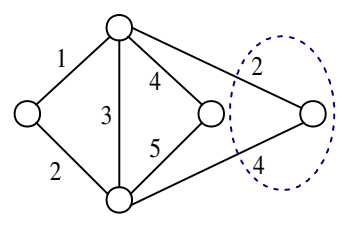

(a) 1-deficient

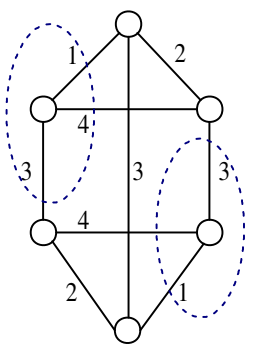

(b) 2-deficient

Fig. 20: The compact wakeup deficiency of graphs

In the compact wakeup scheduling, the compact wakeup deficiency of a graph indicates the minimum number of state transitions. Fig. 20 shows the compact wakeup deficiencies of graphs without valid compact wakeup schedulings shown in Fig. 3. The compact wakeup deficiencies of the vertices in the dashed circles are 1. The compact wakeup deficiency of the graph in Fig. 3 (a) is 1 and the compact wakeup deficiency of the graph in Fig. 3 (b) is 2.

\section{Vi. Performance Evaluation}

In this section, we study the performance of the compact wakeup scheduling of trees and grid graphs, and we also compare our algorithms with the degree-based heuristic in [5] and the contiguous link scheduling in [8]. The performance metrics used in the evaluation are the transient energy consumption and the waiting period. The total energy consumption is an important metric in WSNs, but the energy consumption except the transient energy consumption is the same among the three schemes under identical traffic conditions, so we will only focus on the transient energy consumption. The waiting period is defined as the total time a node stays in the waiting status from the first neighbor waking up to the last neighbor waking up as the node waits for gathering the information from all its neighbors. The waiting period reflects the extra delay caused by the node if it stays in the sleep state for the wakeup of neighbors. 
We adopt the following parameters in our simulation: the transient energy to activate a sensor is $17 \mu J$ [7], a scheduling period $T$ is 10 seconds, and the network operating time is 1 day. In the tree construction of $n$ nodes, the number of children nodes of each sensor is randomly set from 1 to 4 . The root node first determines its children nodes, and then each child node determines its children nodes, and so on and so forth until the total number of nodes in the tree reaches $n$. For each $n, 10$ trees are generated and the average performance over all these trees is reported. In the grid graph construction, we use square grid graphs, where $\mathcal{V}=\mathcal{H}$.

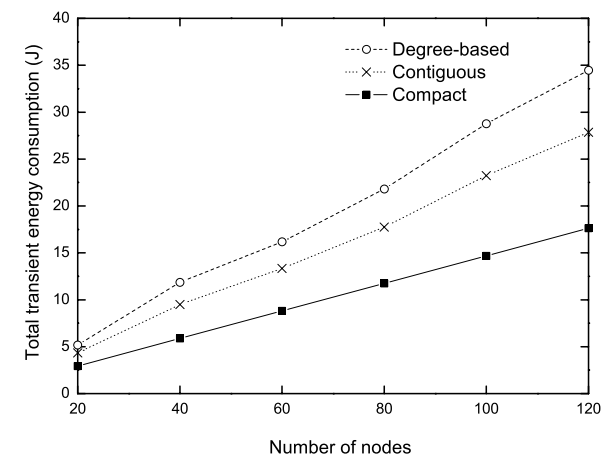

(a) Tree

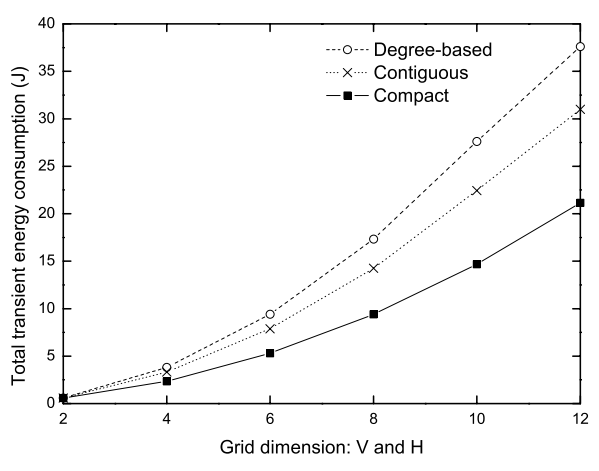

(b) Grid Graph

Fig. 21: Transient energy consumption

Fig. 21 shows the total transient energy consumption of the following schemes: degreebased heuristic (degree-based), contiguous link scheduling (contiguous) and compact wakeup scheduling (compact). In both the tree and grid topologies, the transient energy consumption increases as the number of nodes increases. The energy consumption in the compact wakeup scheduling is the smallest among the three schemes, for the frequency of state transitions is minimized in the scheduling. As shown in Fig. 21 (a), compact wakeup scheduling reduces the energy consumption significantly by approximately $50 \%$ as compared to that in the degree-based heuristic, and about $35 \%$ as compared to that in the contiguous link scheduling.

Fig. 22 shows the total waiting period increases as the number of nodes increases in the degree-based heuristic and contiguous link scheduling, while the waiting period is zero in the compact wakeup scheduling. With smaller waiting periods, it would be faster for nodes to gather the information from their neighbors, thus reducing network delay. 


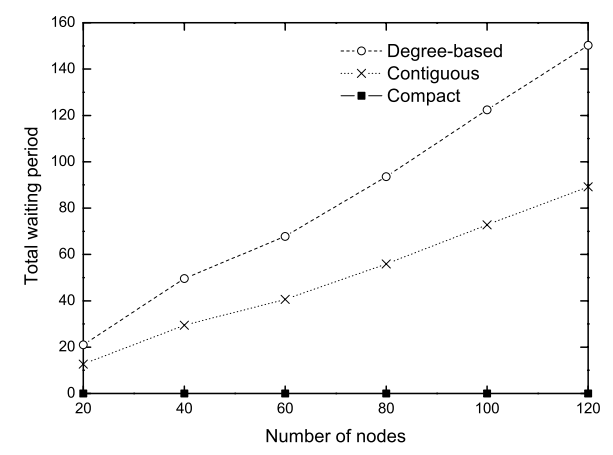

(a) Tree

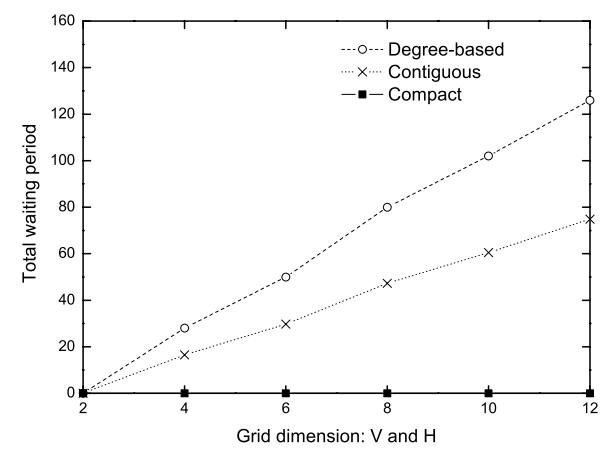

(b) Grid Graph

Fig. 22: Waiting period

We summarize observations from the simulation results as follows: (1) The waiting period of trees and grid graphs with valid compact wakeup scheduling is zero. (2) Compact wakeup scheduling can significantly reduce network delay and energy consumption.

\section{CONCLUSION}

In this paper, we address a new interference-free TDMA wakeup scheduling problem in WSNs, called compact wakeup scheduling. In the scheduling, a node needs to wake up only once to communicate bidirectionally with all its neighbors, thus reducing the time overhead and energy cost in the state transitions. In the case of graphs that have no valid compact wakeup schedulings, we define the compact wakeup deficiency of a graph to extend the scheduling to general graphs. We propose polynomial-time algorithms to achieve the optimum number of time slots assigned in a period for trees and grid graphs. In grid graphs, we point out all the possible coloring patterns and give the lower bound as well as the upper bound of the compact wakeup scheduling. In the process of time slot assignments, both the hidden terminal and exposed terminal problems can be avoided. The simulation results corroborate the theoretical analysis and show the efficiency of compact wakeup scheduling.

In our future work, we will try to obtain efficient algorithms for other kinds of network topologies with valid compact wakeup schedulings. Another interesting problem is to design efficient (i.e. polynomial-time) heuristic algorithms to find an edge-coloring $c$ in a graph $G$ to make the compact wakeup deficiency of coloring $c$ close to $\mathrm{d}(\mathrm{G})$ in general graphs. 


\section{REFERENCES}

[1] W. Ye, J. Heidemann, and D. Estrin, "An energy-efficient MAC protocol for wireless sensor networks," in Proc. of IEEE INFOCOM, 2002.

[2] T. Dam and K. Langendoen, "An adaptive energy-efficient MAC protocol for wireless sensor networks," in Proc. of ACM SenSys, 2003.

[3] Y. Sun, S. Du, O. Gurewitz, and D. B. Johnson, "DW-MAC: a low latency, energy efficient demand-wakeup MAC protocol for wireless sensor networks," in Proc. of ACM MobiHoc, 2008.

[4] A. Keshavarzian, H. Lee, and L. Venkatraman, "Wakeup scheduling in wireless sensor networks," in Proc. of ACM MobiHoc, 2006.

[5] W. Wang, Y. Wang, X. Y. Li, W. Z. Song, and O. Frieder, "Efficient interference-aware TDMA link scheduling for static wireless networks," in Proc. of ACM MobiCom, 2006.

[6] A. Wang, S. Cho, C. Sodini, and A. Chandrakasan, "Energy efficient modulation and MAC for asymmetric RF microsensor systems," in Proc. of the 2001 International Symposium on Low Power Electronics and Design (ISLPED), 2001.

[7] Moteiv Corporation, Tmote Sky Datasheet [Online], Available: http://www.moteiv.com/.

[8] J. Ma, W. Lou, Y. Wu, X. Y. Li, and G. Chen, "Energy efficient TDMA sleep scheduling in wireless sensor networks," in Proc. of IEEE INFOCOM, 2009.

[9] M. Li and Y. Liu, "Underground structure monitoring with wireless sensor networks," in Proc. of ACM/IEEE IPSN, 2007.

[10] W. Song, R. Huang, B. Shirazi, and R. LaHusen, "TreeMAC: Localized TDMA MAC protocol for real-time high-data-rate sensor networks," in Proc. of IEEE PerCom, 2009.

[11] S. Ramanathan and E. L. Lloyd, "Scheduling algorithms for multihop radio networks," IEEE/ACM Transactions on Networking, vol. 1, no. 2, pp. 166-177, 1993.

[12] S. Gandham, M. Dawande, and R. Prakash, "Link scheduling in sensor networks: Distributed edge coloring revisited," in Proc. of IEEE INFOCOM, 2005.

[13] Y. Wu, X. Y. Li, Y. Liu, and W. Lou, "Energy-efficient wake-up scheduling for data collection and aggregation," IEEE Transactions on Parallel and Distributed Systems (TPDS), 2009.

[14] J. Polastre, J. Hill, and D. Culler, "Versatile low power media access for wireless sensor networks," in Proc. of ACM SenSys, 2004.

[15] R. E. Best, Phase-locked Loops: Design, Simulation and Applications. McGraw-Hill, 2003.

[16] V. Bharghavan, A. Demers, S. Shenker, and L. Zhang, "MACAW: a media access protocol for wireless LAN's," in Proc. of ACM SIGCOMM, 1994.

[17] M. Kubale, "Interval edge coloring of a graph with forbidden colors," Discrete Mathematics, vol. 121, no. 1-3, pp. 135-143, 1993.

[18] A. S. Asratian and R. R. Kamalian, "Investigation on interval edge-colorings of graphs," Journal of Combinatorial Theory, Series B, vol. 62, no. 1, pp. 34-43, 1994. 